\title{
Problematics of grounded theory: innovations for developing an increasingly rigorous qualitative method
}

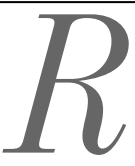

Oualitative Research Copyright (C) 2009

SAGE Publications (Los Angeles, London, New Delhi, Singapore and Washington DC) vol. 9(3) 355-381

\author{
JASON ADAM WASSERMAN \\ Texas Tech University, USA \\ JEFFREY MICHAEL CLAIR \\ University of Alabama at Birmingham, USA \\ KENNETH L. WILSON \\ University of Alabama at Birmingham, USA
}

\begin{abstract}
AвSTRACT Our purpose in this article is to identify and suggest resolution for two core problematics of grounded theory. First, while grounded theory provides transparency to one part of the conceptualization process, where codes emerge directly from the data, it provides no such systematic or transparent way for gaining insight into the conceptual relationships between discovered codes. Producing a grounded theory depends not only on the definition of conceptual pieces, but the delineation of a relationship between at least two of those pieces. Second, the conceptualization process of grounded theory is done in hierarchical fashion, where individual codes emerge from the data but then are used to generate insight into more general concepts and thematic statements. But various works on grounded theory have failed to provide any systematic way of using data specific levels of scale (the codes) to gain insight into more macro levels of scale (concepts and themes). We offer fractal concept analysis as a means of resolving both of these issues. By using a logic structure generator, fractal concept analysis delineates self-similar conceptual frameworks at various levels of abstraction, yielding a method for linking concepts together within and between levels of scale encountered in the grounded theory coding and categorization process. We conclude that this fractal analytic technique can bolster the aims of grounded theory as a formalized and systematic process for generating theory from empirical data.
\end{abstract}

KEYWORDS: ethnography, fractals, grounded theory, homelessness, qualitative methods 


\section{Introduction}

'Problematic' is a word much abused in the academy. Properly utilized, it is not just the intellectual sounding version of the word 'problem,' but rather intended to note ambiguities and things yet unresolved. Our title is something of a tribute to Neil Smelser's Problematics of Sociology, a work premised on the idea that disciplinary bickering over macro and micro approaches proceeds under a cloud of ambiguity about what those really are. Similarly, for the varying grounded theory approaches offered and vigorously defended, there remain glaring ambiguities about the theory building process.

Our purpose in this article is to identify and suggest resolution for two core problematics of grounded theory. First, grounded theory provides transparency to one part of the conceptualization process, where concepts, initially in the form of codes, 'emerge' directly from the data in a process brilliantly designed to facilitate the development of the researcher's conceptual insight. But then, at the crucial point where real theory begins, it provides no such systematic or transparent way for gaining insight into the conceptual relationships between emergent concepts. Producing theory depends not only on the definition of conceptual pieces, but the delineation of a relationship between at least two of those pieces. For example, we can delineate a concept of poverty and a concept of health status, but we have no real theory until we gain insight into how poverty and health interweave each other. As building theory is the central purpose of grounded theory, the failure to provide facilitation for conceptual relationships, as it does for conceptual pieces, ought to be an important concern.

Second, the conceptualization process of grounded theory is done in hierarchical fashion, where individual codes emerge from the data but then are used to generate insight into more general concepts and thematic statements. But various works on grounded theory have failed to provide any systematic way of using data specific levels of scale (the codes) to gain insight into concepts at broader levels of scale (categories and ultimately themes).

Along with discussing these two problematics of grounded theory, we offer a fresh approach for resolving them, which we call fractal concept analysis. ${ }^{1}$ As self-organized systems replicated at various levels of scale, the fundamental nature of fractals suggests a clear benefit to grounded theory. Such a technique allows us to assemble multiple emergent concepts into conceptual structures and to systematically work between data-specific and broader levels of scale. After discussing this analytic technique in more detail, we offer a basic example from our ethnographic homeless research to illustrate its success. While we directly address grounded theory, fractal concept analysis speaks more broadly to the empirical problems encountered in an array of qualitative approaches, many of which are 'grounded' in the data in one way or another.

Additionally, while social science often is consumed with epistemic disagreements between positivists and interpretivists, a dichotomy too often 
misunderstood as synonymous with the quantitative-qualitative divide, we show how fractal concept analysis can avoid the trappings of naïve positivism without falling into nihilistic relativism. That is, we can make the empirical processes of grounded theory more rigorous without eliminating the notion of conceptual analysis as multi-dimensional, but also without falling into the 'anything goes' mentality of extreme postmodernism (see also Rennie 1998, for a discussion of grounded theory as a combination of abductive and inductive processes which steers between realism and relativism).

\section{Grounded theory}

\section{A SORDID PAST}

There is a bit of sociological folklore about C. Wright Mills that we have heard in various incarnations throughout the years. We will paraphrase the story like this:

Upon publication of The Power Elite, a sociologist friend of Mills published a scathing critique of his work. The average scholar would be heart-broken; these are the kinds of things that end longstanding friendships in academia. Being an established force in the discipline, Mills certainly would have been in a position to do battle. Instead he replied with a letter containing only one sentence: 'How can I make it better?'

We do not know if this story is true, but clearly it is instructive. To be actively intellectual means to resist circling one's wagons, to instead work toward new ideas all of the time. This is easier said than done, but certainly is something for which to strive. Grounded theory often is hotly contested with some past discussions being rather nasty. ${ }^{2}$ This is a shame.

We might easily have ignored this sordid past of grounded theory, but as we hope to offer an innovative work, it is appropriate to first identify and counter things that stand in the way. We appeal to those who wish to be creative rather than dogmatic on recognition that the basis of intellectual life is that no proposition is beyond critique, no idea beyond improvement. We join in spirit, if not exact opinion, Bryant (2002), Charmaz (2006), Clarke (2005), and many scholars from different disciplines across the globe in feeling that the basics of the grounded theory framework can be merged with new analytic techniques that will continue to generate creatively diverse studies, acknowledging that how the grounded theory guidelines and assumptions are used is not a neutral process (see also Bryant and Charmaz, 2007).

\section{THE GROUNDED THEORY TECHNIQUE}

Certainly grounded theory as a whole represents not just a specific analytic schematic, but more generally an epistemic frame of mind. Where we refer to it as a 'technique' however, we wish to indicate its specific analytic processes as laid out by its founders and proponents because it is there that the problematics 
we identify emerge most clearly. The general epistemic orientation of grounded theory really is widely shared in both the natural and social sciences, emerging wherever data are allowed to directly generate knowledge, rather than used to verify hypotheses. Ethnographers, for example, commonly utilize a grounded episteme even when not utilizing the specific coding and conceptualization techniques of grounded theory proper. Additionally, while Glaser (2002) suggests that grounded theory generally overcomes distinctions between qualitative and quantitative methods, as an analytic process it primarily has been utilized to generate insights from qualitative data. We therefore focus our efforts at grounded theory as a qualitative method, but certainly do not deny its broader possibilities.

In so far as we address problematics found within the most basic coding and conceptualization processes of grounded theory, our treatment of it will attempt to focus on the root description found in Glaser and Strauss (1967). We note significant developments where appropriate, but tracing out the variations that descended from this seminal work is beyond the scope of this article (for this, see Charmaz, 2000; Rennie, 1996). However, it is our estimation that fractal concept analysis will fit with any variation, although it will be underpinned by different assumptions, which may have an effect on the ultimate interpretation of the conceptual products. Finally, our description of grounded theory will be common knowledge to many readers, but its rampant abuse suggests that assuming understanding or consensus is not warranted (see especially Glaser [2002] for an account of the improper uses of grounded theory).

Grounded theory was 'discovered' by Glaser and Strauss $(1965,1968)$ during their studies of death and dying in hospitals, first detailed in their foundational work, The Discovery of Grounded Theory (1967), and then further specified in Glaser (1978). ${ }^{3}$ It essentially is a process of coding data and then grouping those codes into concepts in an increasingly hierarchical fashion (although we should note that 'codes' also are concepts which denote particular parts of the data). Ultimately, theoretical models emerge where concepts are arranged into theoretical propositions. Unlike typical hypothesis testing, which begins with the development of hypotheses that are tested with subsequently collected data, in grounded theory everything begins with the data.

Furthermore, rather than employing the standard order of data collection then data analysis, in grounded theory data collection and analysis is dynamic and multi-layered. That is, motivated by the notion that sociological concepts should be an organic, integral part of social life rather than something artificially attached by remote experts, data is collected and then analysed and then more data is collected and analyzed in order to pursue emerging themes from the first wave of analysis. This cyclical process is repeated until there is no new emergent conceptual information, a point that has been called 'saturation' (Glaser and Strauss, 1967). Conceptual themes are pursued intentionally by focusing or altering questions in subsequent interviews and by theoretically sampling new respondents. 
Initial coding is open-ended and done 'line by line.' ${ }^{4}$ This essentially means breaking down the data and identifying concepts embedded within individual statements. As line by line coding proceeds, recurring concepts will illustrate 'focused codes' (Charmaz, 2006). Focused codes are directed, representing what the researcher feels most accurately explain the recurring concepts that are beginning to emerge from the data.

In grounded theory, data driven themes emerge through a process of 'constant comparison' (Glaser and Strauss, 1967). As it literally indicates, this means that everything is compared with everything and all of the time. Statements within an interview are compared with each other and with other interviews, new data are compared with older data, conceptual categories derived from some data are compared with other data directly and with the conceptual categories derived from that data, and so on (Charmaz, 2006; see also Clarke [2005] for her unique focus on situational, social and positional mapping processes). While grounded theory often is understood as a buildingup of concepts from the data, the process of constant comparison brings dataspecific codes and broader concepts into an insight generating dialogue, as opposed to a simple grouping process.

As focused codes and broader concepts emerge from the data one must remember that they do not become a priori concepts for subsequently collected data. Instead, grounded theory deals with discrepancies between new data and previous concepts by modifying the conceptual scheme with synthesis, rather than dismissing or explaining away non-fitting data or 'forcing' that data to fit (Glaser, 1992). There is no such thing as an outlier in grounded theory, in the sense that no data is dismissed from investigation because of non-central tendencies. Rather, concepts, and the larger conceptual scheme, are constantly improved by synthesizing new data or new analysis of older data. ${ }^{5}$ For those committed to more typical theory building strategies where concepts are thought of as mere abstractions of data, such a process seems to only generate uselessly abstract concepts lacking the ability to predict new social outcomes. This would underestimate the value of developing concepts intimately connected with, and responsive to, actually lived social life.

As focused coding proceeds, the researcher engages in the memo-making process. ${ }^{6}$ Memo writing allows the researcher to flesh out emergent concepts. Memos are written in narrative form and allow the researchers to expound on emergent themes while building linkages between concepts. Through this process one is able to elevate significant codes into categories. By continually comparing data with categories in memos, categories begin to fill out and can be further elaborated, advancing theory development throughout the whole process. Through theoretical sampling, new data are sought, and with the procedure of constant comparison, one eventually moves toward developing a theoretical framework. While writing up a manuscript draft, new gaps are identified and concepts further refined. As the grounded theorist finally turns to the existing literature, the whole piece is reworked, which culminates in theory construction (Charmaz, 2001). 
In the end, through all these procedures, grounded theorists suggest that we get integrated theory. However, exactly how theory leaps out of the data through this process will still seem ambiguous to the reader and rightly so. In other words, while it may be clear how the technique promotes insight in the development of initial codes, the ones that are explicit in the data, it is not clear exactly how this promotes insight into conceptual structures, and thereby leaves one with the impression that the latter may be based merely on the musings of the individual researcher. Glaser (1978: 72) attempts to address this by delineating theoretical coding as separate from but analogous to substantive coding, but he admits, 'theoretical codes are always implicit' and ultimately leaves it unclear just how they can be surely explicated. However, his suggestion of 18 commonly discovered theoretical coding families demonstrates a need to address this ambiguity. We fill this need with fractal concept analysis, which can encapsulate Glaser's (1978) suggested theoretical coding families in a way that explicates a logic structure for theory building. We later return to Glaser's theoretical coding families in comparison to the analytic technique we offer here.

\section{ASSESSING GROUNDED THEORY}

In a refreshingly brazen way, Glaser and Strauss (1967) asserted grounded theory as a formal way to develop theory. Kuhn's (1962) Structure of Scientific Revolutions had been published five years earlier, but sociology was not yet fully in the grip of the postmodern critique. While they did not intend to suggest that grounded theory was deductive, by today's standards the claims of Glaser and Strauss that grounded theory is a technique of discovering concepts that are in the data and indeed that they had themselves 'discovered' the technique, may seem exceedingly positivist. Many are skeptical about the extent to which grounded theory successfully systematizes empirical observation through its analytic processes. After all, coding and categorizing are still in the hands of the individual researcher. But assessing grounded theory on whether emergent concepts have truth correspondence to the actual world not only is abysmal, it is unfair (see also Carrithers, 1990). Quantitative techniques have not met that challenge, yet widely are held as formal and even as approximating natural science. The fair test of grounded theory concerns whether its particular analytic processes improve observation and it ultimately is on these grounds that integrating fractal concept analysis moves toward a more rigorous way of analyzing data.

It is commonly, though not universally, held that researcher bias is antithetical to rigorous science. While many epistemologists contend that human biases simply are part of science either because they cannot or should not be controlled for, those doing research mostly attempt to control for bias usually either by eliminating it with various methodological controls - the approach more popular in quantitative research - or by indulging bias to make it explicit and transparent - the approach more popular in qualitative research. Fundamentally, bias is a problem primarily generated in the act of empirical 
observation. Possible confounding factors might include the researcher's political and social values or personal conflicts and self-interest, but these ultimately are concerns because they potentially cloud observation. The development of science is the accumulation of techniques that control for the unreliability of raw empirical observation. This is why qualitative research has fallen behind in the estimation of the increasingly quantitatively oriented social sciences; most qualitative research remains wholly and unapologetically dependent upon raw observation.

Those who criticize the scientistic claims of quantitative techniques are quite correct, however, on at least one account. That is, quantitative methods also are dependent on observation and therefore subject to the same fundamental critique, though we may convincingly say that on the whole the quantitative camp has done a better job of responding. Still, those crunching the numbers often do conveniently forget that statistical work functions simply by turning empirical observations into numerical expressions, but still are observations. So the ultimate question for both quantitative and qualitative methods is the same: can observation be trusted or are we doomed to the nihilism of extreme postmodernists? Quantitative measurement approaches (at least those done rigorously) indeed suggest that observation can be made better or worse, that is, more or less reliable.

Take for example that as researchers we decide to assess hospital treatments as correlated with various other things. We could sit at the hospital and count the number of times a given person comes in over the course of a year. But observing entrances into the hospital may not capture conceptually what we are actually interested in. A person could simply be visiting a sick relative. So we should modify our observational parameter to eliminate this factor. Perhaps we can use medical records to identify the number of treatments that a person received. This will rule out all visitors. However, what counts as treatment? If a person seeks treatment for a deep cut and then returns to have the stitches removed, does this count as one treatment or two? This all depends on what our suspected correlates of interest are, but for now, let us say we wish it to count such a case as one treatment. We might therefore additionally modify our observational scheme to control for multiple visits for the treatment of single conditions. In each step we improve our observations by controlling for the confounding factors of raw empiricism.

Additionally, we may change our hospital treatment research altogether and decide to ask people themselves how many times they have been to the hospital to deal with medical problems. In doing so we have controlled for our own bias as researchers, but now rely on the observations of each respondent. Specifically, we are faced with respondent recall bias. If we ask about hospital treatments over the last 10 years, the self-observations may be rather fuzzy. So we then would likely modify our recall frame to cover only one year. Perhaps we have belabored the point, but it is as important as it is simple. Researcher bias can be attenuated; empirical observation can be made better or worse. 
While qualitative approaches in general may not have made as much progress as quantitative approaches with what we might call the problem of raw observation, grounded theory has provided a comparatively high standard. Not only does grounded theory use social science data, which is fundamentally observational, but its conceptualization techniques essentially instruct a process of making observations about data, or we might say, observations about observations.

Certainly the most admirable thing about Glaser and Strauss (1967) was that they took a position that qualitative researchers at the time were shying away from and now seem to have abandoned entirely. ${ }^{7}$ That is, they courageously asserted that qualitative research can be made more rigorous, that its observations can be made more reliable. While people have varying opinions about exactly how successful grounded theory is on this account, the comparative processes and other conceptualization techniques (e.g. hierarchical coding processes) make clear improvements on unchecked empiricism. Just as we improved our observational scheme in the above hypothetical, grounded theory compels us to improve our conceptual scheme (i.e. our grouped observations of the data) when new observations clash with previously emergent conceptions.

But how does grounded theory fare with regard to developing theory from observed concepts? Quantitative approaches are able to side-step this problem because they can support or discredit any theory via the verification or disconfirmation of hypotheses; essentially they are able to leave it up to the theorist, suggesting that scientists can hypothesize whatever they want so long as they test their hypotheses. Philosophy of science has essentially endorsed this move by calling the process through which scientists intuit theoretical ideas the "psychological problem' (Hume, 1739 [1978]; Popper, 2002; Wundt, 1973). While grounded theorists have taken this problem head-on, the process offered for theory building is the same process used for developing concepts (see Glaser's [1978] discussion of 'theoretical coding'). Just as postulated concepts have to enter into a dialogue with the raw observations until confidence emerges, so also do theoretical structures have to dialogue with both concepts and raw observations until confidence emerges. But without a unique process for the latter, it remains unclear whether grounded theory provides an actual improvement over conventional theory building practices that allow theorists to use their intuitions to do what may be essentially the same thing. In other words, grounded theory clearly improves the conceptualization process where specific concepts (i.e. codes) emerge from the data, but the assumed extension of this technique to assembling concepts into theory is unsatisfying. The data illustrate the codes, but there is no explicated technique that convincingly illustrates how multiple codes fit together in a logical relation or in a broader conceptual grouping. So while grounded theorists have spent much time on coding, they leave unclear just how the logical relation of multiple concepts, that is theory, jumps out of the data through this coding process, even when 
supplementing it with 'constant comparison'. To address this, we turn to fractal concept analysis, which can supply the needed logic structure. We note at the outset that we need make no claim that the world is fractal-like. That is, the truth correspondence of fractals to reality is not a good standard for judging the utility of our analytic suggestion. Linear logic can (and does) elucidate all sorts of knowledge about the world, but few would say that everything in the world is linear. Similarly, we simply have to show that the inter-scale ontological relationships suggested by fractals have utility for grounded theory analysis.

\section{Fractal concept analysis}

\section{A SOCIAL SCIENCE INTRODUCTION TO FRACTALS}

Fractals are structures that are self-similar at various levels of scale. Mandelbrot (1982) made the initial discovery of fractal patterns by noting cotton prices, which were thought to be chaotic (i.e. functions of unpredictable phenomena like climate), actually repeat a similar pattern across multiple levels of scale (measured as time; see Figure 1). That is, regardless of whether one examined the ebbs and flows of cotton prices across several weeks, several months, or several years, the 'lightning bolt' pattern is repeated.

For mathematics, fractals can be expressed as a basic equation for which the output of an initial run is used as the input for a subsequent one. This process is called iteration. When this process is repeated in a continuous feedback loop, and the data plotted, fractal pictures emerge. The basic equation with no real data is called a 'generator'; it essentially denotes the form that each run will replicate (for Mandelbrot, this is the 'lightning bolt' structure). Figure 2 depicts a fractal with a ' $V$ ' shaped generator. Note that the ' $V$ ' shape is replicated first twice, and then four times, as the plotted equation is put through several iterations. While the process of moving toward more complexity is called iteration, the reverse process is called catiteration. So while we may work forward in the fractal and get more branching ' $V$ ' shaped patterns with each iteration, we may also work backward (catiteration) toward the initial ' $V$ '.

Despite preference for quantitative methods and the widespread recognition of the limited explanatory power of linear statistics, social science has been relatively slow to explore, much less utilize, fractal approaches. Abbott (2001) is a notable exception. In a conceptual analysis, he notes the history of the social sciences themselves can be understood as fractal iteration of a binary generator based on the Kantian distinction of pure and practical reason.

Following Abbott, Salzinger (2004) offers a less esoteric example of fractal patterns of gendered identities in the medical professions. ${ }^{8}$ The branching pattern of this conceptual fractal (Figure 3 ) is similar to the branching pattern in the plotted equation of Figure 2. Using fractals, we can depict the way in which gender constructions play out in the medical professions with more complexity, and thus more accuracy, than linear conceptualizations can allow. For example, we can understand that the subspecialty of pediatrics is relatively 

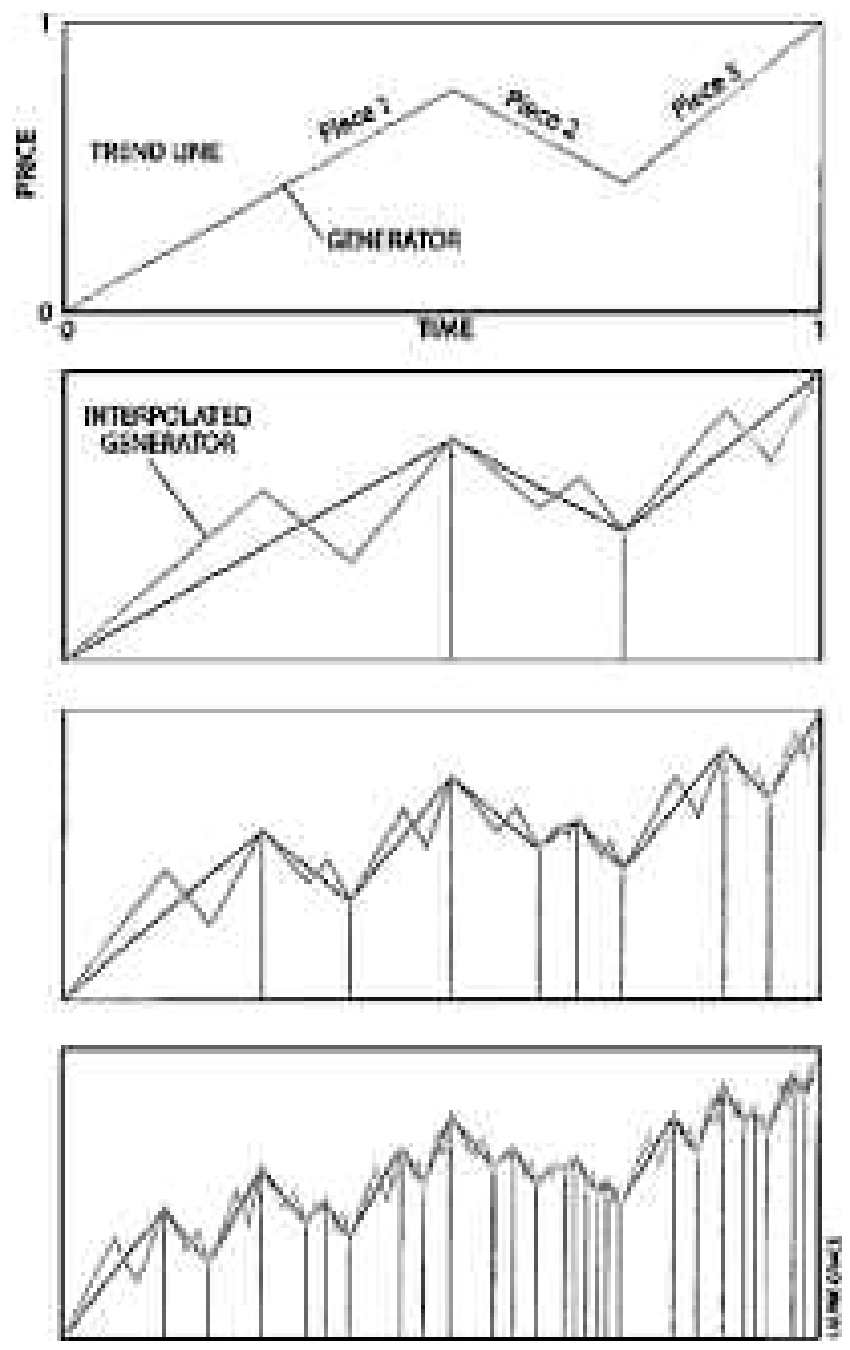

Figure 1 Fractal seed (trend line) and generator (3-piece lightning bolt shape) in Mandelbrot's (2004) analysis of price charts

From: http://www.elliottwave.com/education/SciAmerican/Mandelbrot_Article2.htm

feminine when compared with surgery, but that both of these are members of the physician profession, which is relatively masculine when compared with the profession of nursing. Linear approaches cannot attend to these types of complexities (statistically or conceptually), but most often are limited to singular categorical understandings.

Quantitative social science approaches attempt to attenuate researcher bias by sacrificing conceptual depth for specification. As the example in Figure 3 shows, fractals hold the promise for systematizing observation without sacrificing complexity. Science relies on logic to find systematic regularities among observations. Linear logic applies well to natural phenomena, for example those of physics, but clearly falls short for human sciences, since people are not just automatons, but rather possess the capacity for intention and will. Therefore, when assessed by linear logic, human behavior often appears 


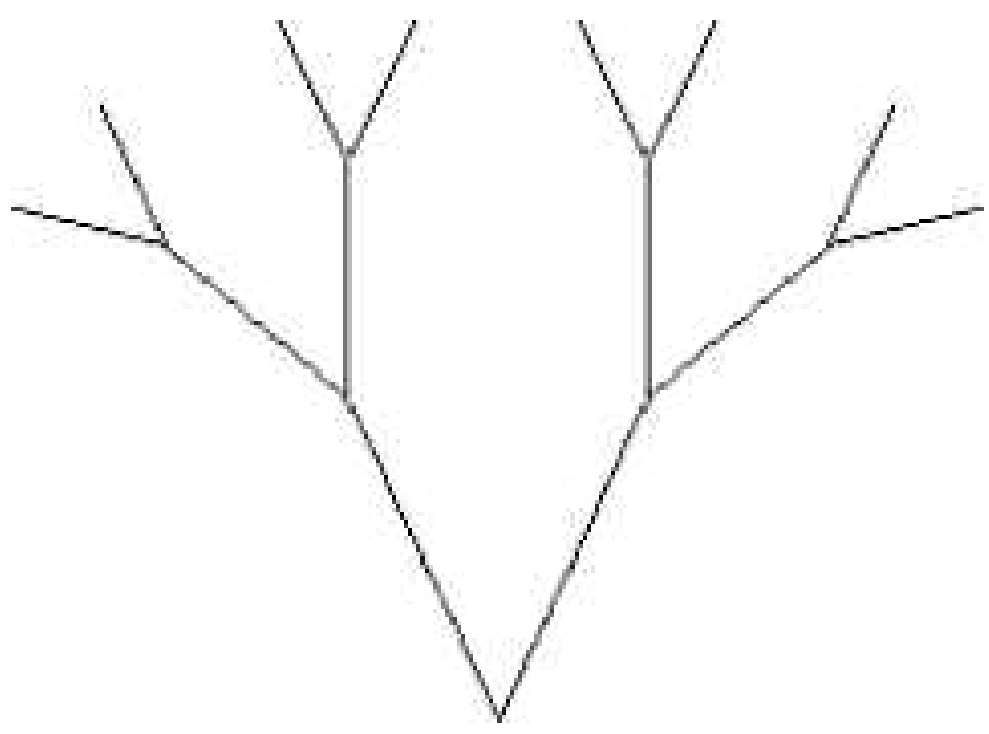

Figure 2 Data plot of ' $V$ ' generator iterated two times

Screen capture from: http://id.mind.net/ zona/mmts/geometrySection/fractals/tree/ treeFractal.html

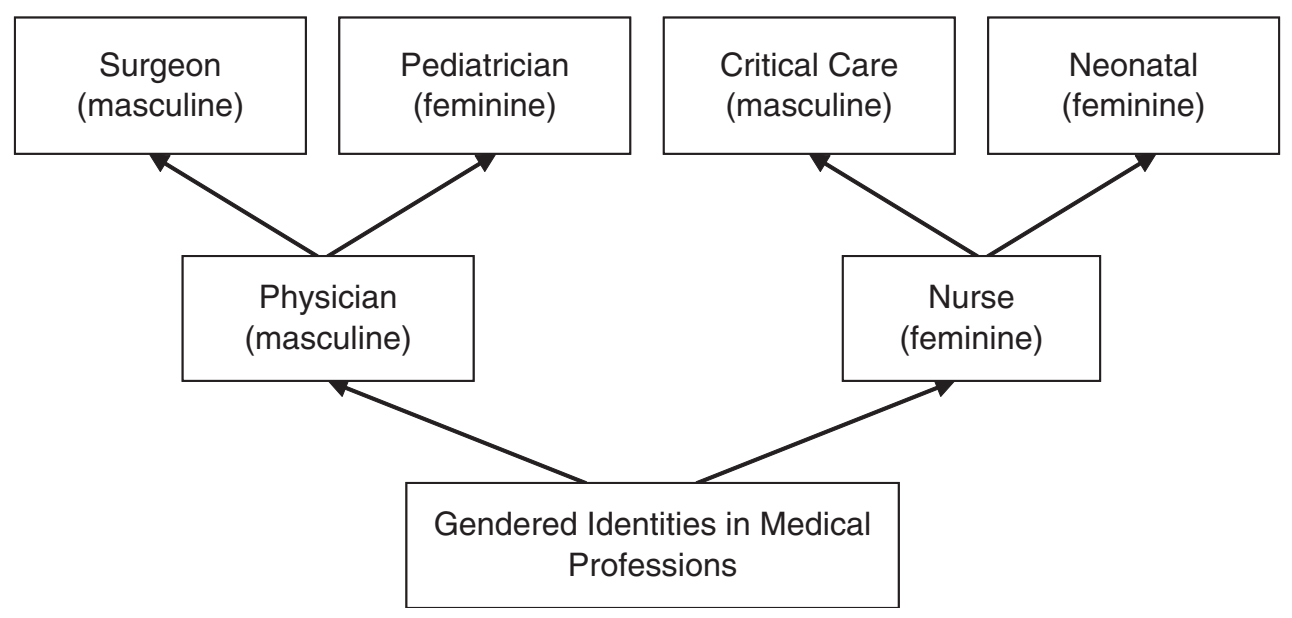

FiguRe 3 Binary gender generator iterated two times for medical professions (derived from Salzinger 2004 and the Men in Nursing Survey 2004)

chaotic. While certainly providing insight, linear methods routinely peak at explaining only 50 percent of the variance in a sample, and that on a very good day. While we tend to chalk the large portions of unexplained variance up to the fact that human behavior is rather unpredictable, we might also conclude that linear logic simply is insufficient for understanding something as complex as human life. ${ }^{9}$

Fractals have the capacity to incorporate multiple styles of logic organized into generators. For example, a binary generator organizes the fractals found in Abbott (2001) and Salzinger (2004; see Figure 3). These binary generators not 


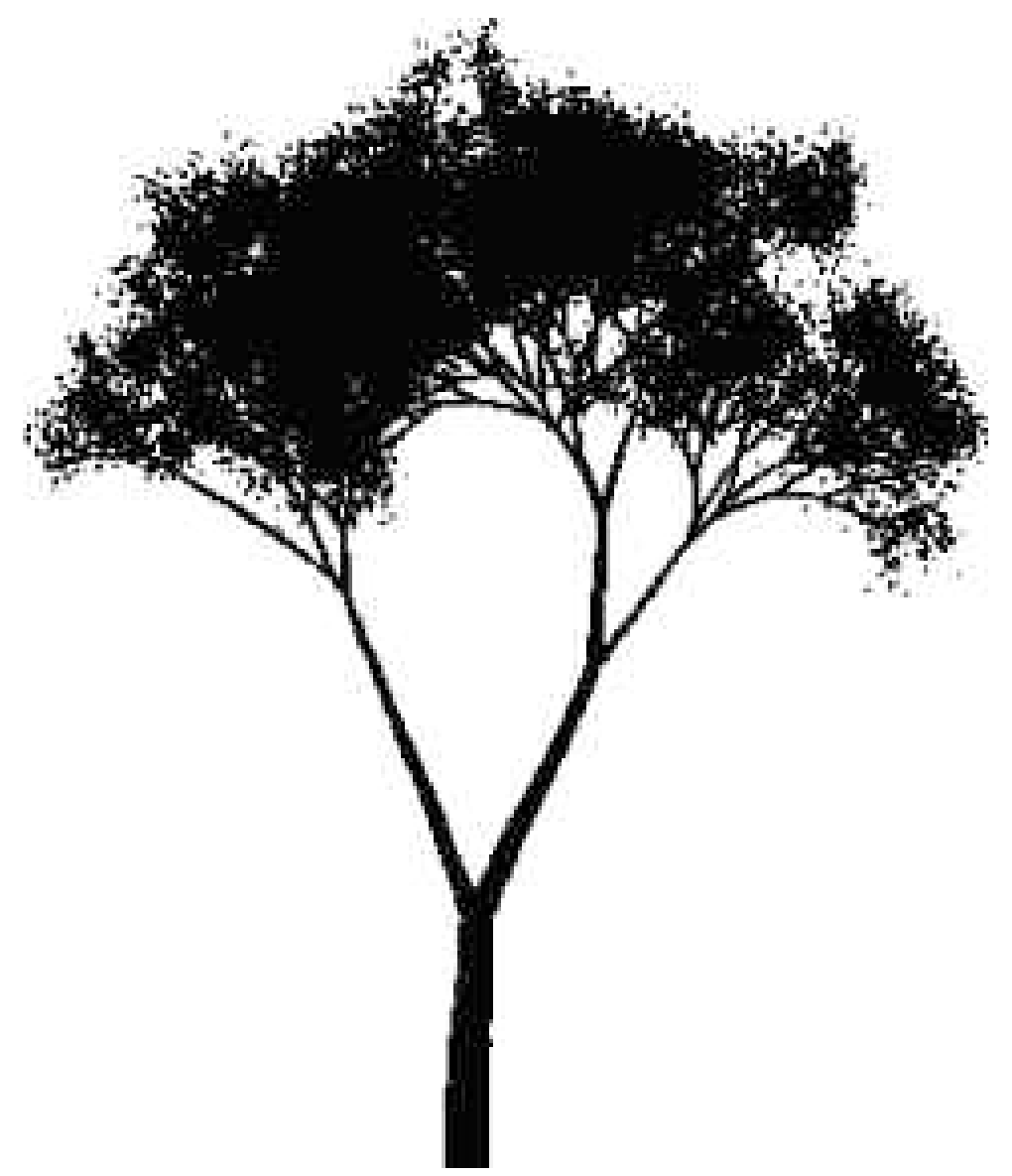

FIgURE 4 Fractal tree using more complex generator

Screen capture from: http://www.webcalc.net/calc/0467.php

only specify independent kinds of concepts, but also unique styles of hypothesizing interrelationships among concepts on each side of the binary split. This begs the question about what other sorts of generators we might find useful in analysis of human phenomenon since the world is not just a series of binary oppositions.

Returning to mathematical fractals for a moment, the branching pattern of the ' $\mathrm{V}$ ' shaped generator in Figure 2 has been described as a fractal tree, but certainly it does not look like any trees we can see in nature. However, as Figure 4 shows, a slightly more complex fractal generator yields a picture that looks much more like a tree. This is more than analogy; it illustrates the influence of the fractal generator on the observational process. This should lead us to suspect that a more complex conceptual generator can give a more accurate depiction of social phenomena. Next we present just such a fractal generator, one organized according to a more human cognition (relative to linear or binary logic systems), and therefore yielding more accurate depictions of human phenomenon. 
TABLE 1. MIC generator

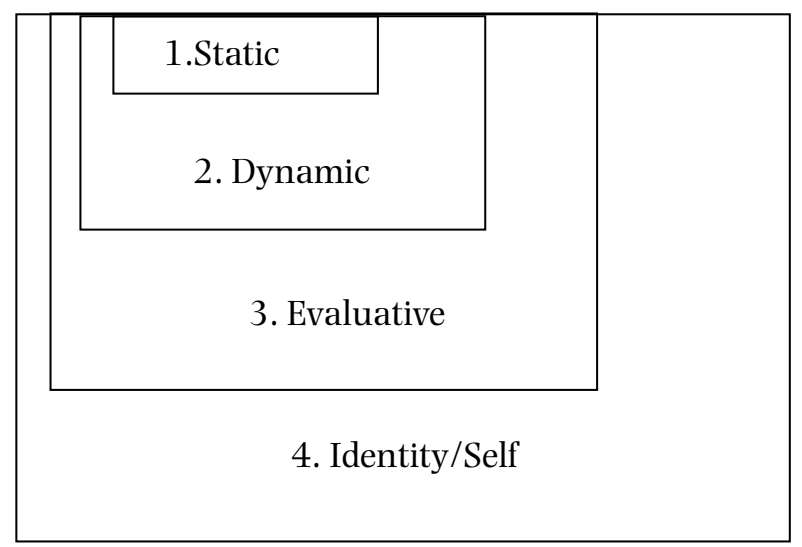

THE MIC GENERATOR

Multilevel Integrated Cognition (MIC) (Wilson and Lowndes, 2004) employs four basic ontological categories. These are (1) static, (2) dynamic, (3) evaluative, and (4) self/identity (Wilson and Lowndes, 2004: Table 1; also see Wasserman and Wilson, 2008, for application of the MIC categories to analyzing visual media and teaching). These four levels have the capacity to organize complexity far beyond binary generators. They not only delineate ontological categories with uniquely formed concepts, but also the relations between concepts within each level (i.e., static theoretical relationships, dynamic theoretical relationships, etc.) and, finally, the relations between levels (organized in a hierarchical, Guttman-like system of levels), all put together in a holistic picture of the human world. As such, these concepts possess particular inherent interconnections while at the same time avoiding limitations of substantive conceptual systems (e.g. Glaser's theoretical coding families).

We first define each of the four levels and then illustrate the interconnectedness of the whole system. In doing so it will become clear how MIC, as a meta-structure, benefits grounded theory by systematically linking concepts together into theoretical structures. ${ }^{10}$ This answers the first core problematic identified at the outset. Second, we move beyond MIC as an ontological framework discerning relationships between conceptual pieces, toward its use as a fractal generator which illuminates its contributions to working across multiple levels of scale, formalizing the theory building process (for fractal applications of MIC in visual art, see Wilson et al., 2009; and in music, see Wilson et al., 2008). This answers the second core problematic.

MIC identifies four kinds of concepts. The static level refers simply to objects which can be delineated as things unto themselves, elements, things that are this but not that. But not all observed phenomena are able to be broken down to the static level. Dynamic concepts refer to action, an ontologically different kind of thing than the elemental approach of the static level. Where we 
attempt to wrestle dynamics into the realm of statics, we are left with insufficient conceptualizations. In Zeno's Paradox of Dichotomy, for example, the impossibility of moving from point A to point B is logically validated on the premise that there are an infinite number of midpoints at which one must arrive before reaching $\mathrm{B}$. This paradox results from an ontological disposition confined to an atomistic position that cannot fully define the dynamic quality of nature. The concept of movement is not a series of leaps from point to point, but a fully dynamic process, for which static objects, like Zeno's midpoints, cannot account. Thus, we must retain the vision of dynamic concepts as ontologically different from static concepts.

The evaluative level encompasses judgments, values, and feelings. These are ontologically distinct from levels one and two, since nothing about elements or processes inherently implies a value embedded in them or a feeling that they evoke. That a flower has leaves, petals, a stem, grows, and bends toward light says nothing about whether it is beautiful or ugly. In philosophy, we can see this delineation reflected, among other places, in Hume's (1739 [1978]) notion that we cannot derive 'ought' from 'is'. As such, a well-formed observational framework for human phenomenon should recognize an ontological distinction between level three and the others. Human life, and also that of other animals, is keenly punctuated by feelings and values (although we might say the latter are particularly human). These values and feelings are what essentially separate animals from plants, which have elements and undergo processes, but do not experience level three.

Level four extends further into the human aspect of knowledge by recognizing the notion of unique identity. This includes conceptual designations, which humans can ascribe to various things they observe, such as the way the concept 'rose' usually denotes a complex of elements (leaves, stem), processes (growth, photosynthesis), and valuative judgments (beauty). Furthermore, level four encompasses concepts of self, which are distinctly human identities. We are compelled to designate level four as a distinct ontological category in our framework because at a particular point human experience transcends that of animals. ${ }^{11}$ The very essence of being animalistic is to be guided by sense as opposed to thought. To be sure, humans often are guided by feeling concepts born from sensory experience (level three). But in reflexive thought, human beings extend their existence beyond an animal existence into a realm purely human. At the identity/self level are concepts of agency, uniqueness, and creativity.

At this point, one will notice that Glaser's (1978) theoretical coding families correspond to our four ontological levels, especially where he designates an identity-self family. However, a comparison of the MIC generator and Glaser's coding families shows the difference between them. The MIC generator identifies ontological distinctions and thus a logic structure. As such, Glaser's theoretical coding families could be folded into our four-level ontological schema and while this ontological schema was established independently of Glaser's 
coding families, we might have arrived at them by catiterating from them. For example, Glaser's (1978: 75) 'Dimension Family' contains 'elements, division, piece of, properties of, slice, sector, portion, segment, part, etc.' From our ontological standpoint, we would fold these largely into the static level.

Furthermore, the MIC generator does not express independent categories as does Glaser (1978), but rather logically interrelated notions in a system where the higher levels always contain the lower levels. Humans can be said to incorporate all four levels, animals levels one through three, plants levels one and two, and minerals level one. Thus, as we will see in the next section, applying the MIC generator to the study of humans and human observation, yields logical structure to ontologically distinct observations, thereby building a conceptual model, not just a theoretical taxonomy.

\section{Resolving the problematics of grounded theory}

In this section we discuss and exemplify how the MIC fractal generator can resolve the two core problematics of grounded theory, at least for its application to social science data (since MIC is a human logic structure, non-human data would require alternative generators). To achieve this, we first offer an excerpt of ethnographic fieldnotes from research on the street homeless. This will illustrate the way in which the MIC generator can provide systematic linkage between concepts such that they form a conceptual model at a data specific level of scale. Second, we take concepts generated from that same research project and catiterate them back toward more macro concept structures, showing how the MIC concept structure is replicated across levels of scale. Fractal catiteration operates fundamentally like other versions of grounded theory, but with fractals, we can see the replication of the same logic structure each time we abstract. Fractal concept analysis therefore can be seen as a formal mechanism for identifying and validating macro level conceptualizations derived from micro level observational data.

\section{PROBLEMATIC ONE: CONCEPTUAL RELATIONSHIPS}

The right column of Table 2 is an excerpt from fieldnotes generated during an ethnographic research project with the street homeless. The basic coding of this excerpt is shown in the middle column and the left-hand column shows how they are organized by the four level schema of MIC (it is important to note that the left hand column is not a separate coding process, but rather the identification of ontological distinction among the substantive codes themselves). Substantively speaking, codes are shorthand conceptual references for the comparatively lengthy narrative of fieldnotes. Coding not only functions to make concepts logistically manageable, it also brings concepts into acute focus when they otherwise would remain diffuse in the narrative structure of the data. Moreover, it is important that the substantive content of the codes in the middle column are precisely the same codes we would have derived without 
TABLE 2. MIC coding of fieldnotes

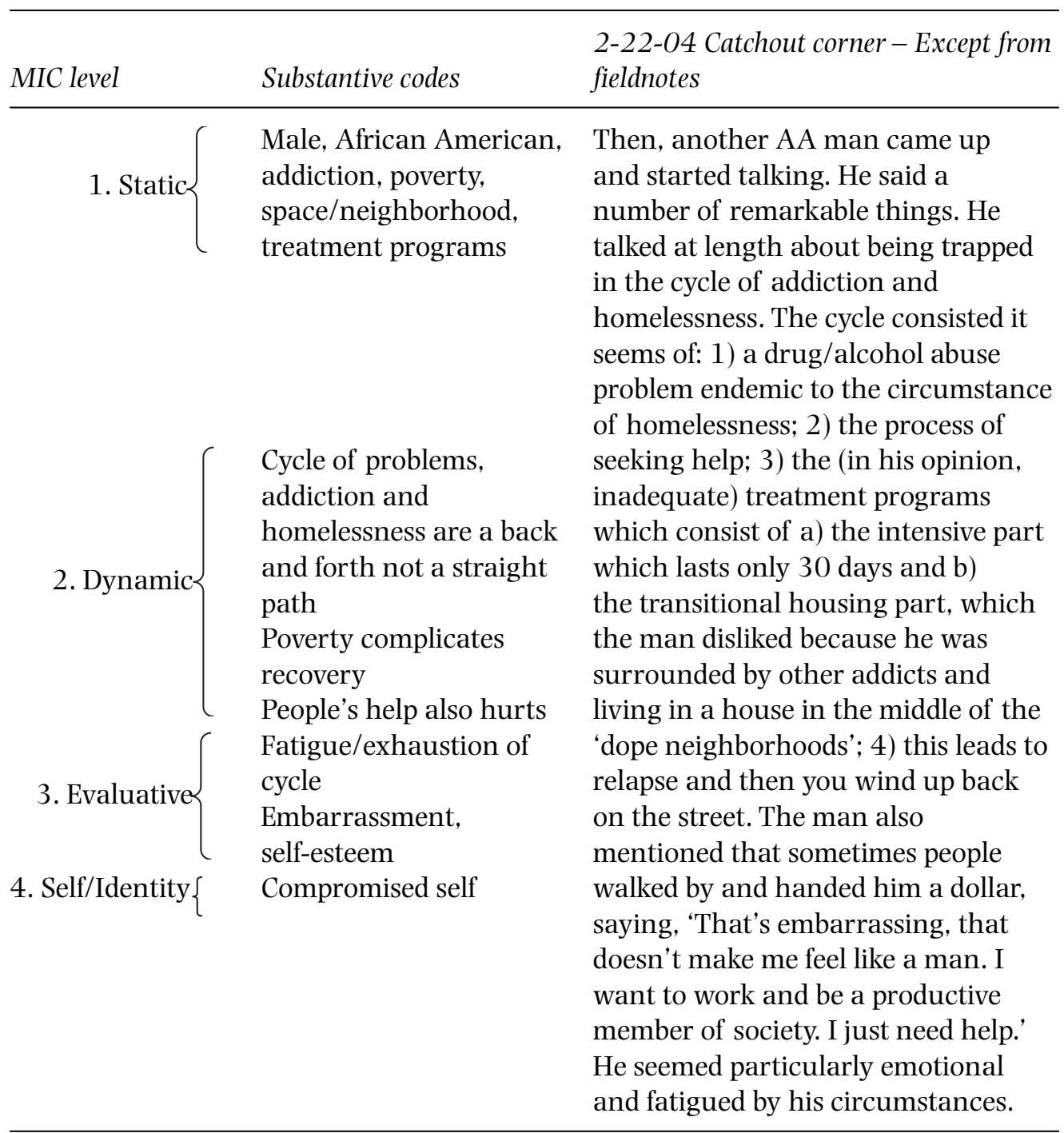

any ontological input from the MIC generator, a fact that will become important later.

Rather than alter the conceptual substance, interpolating the MIC generator onto the codes illustrates first that they are not of like kind (some are static elements, some are processes or relationships, etc.) and second they are interconnected in a logical relation. From this excerpt we can see two conceptual models: 1) the elements of poverty and addiction (static) engaged in cyclical interaction (dynamic) produce the emotional fatigue and exhaustion of continued homelessness (evaluative) and 2) people's actions of giving money (dynamic) produce a feeling of embarrassment (evaluative) which results in a diminished concept of self (self/identity).

Two additional points of clarification are needed. First, our short excerpt here is simple enough so that most people can intuitively make such connections 
between concepts. The tendency may be to question the significance of the revelation of these rather simple conceptual models. However, our point has been to illustrate the systematic interconnection that the MIC generator yields, rather than any revolutionary insights into conceptual models of homelessness. The revelation here is methodological: conceptual model building, like the initial coding of conceptual pieces, can be guided by a transparent process. Furthermore, this becomes particularly important as data becomes more massive, esoteric, or foreign to the established dispositions of the researcher, where raw intuition is less reliable than it is for our simplistic example here.

Second, the reader will note that concepts were not explicated for each of the four levels in each model. This is a shortcoming of the fieldnote narrative, which is characterized by comparatively unstructured observation and further highlights the benefit of employing MIC. The logic structure provided by interpolating the MIC generator suggests things we may have missed. For the second conceptual model, these are easy to specify. That is, implicit static elements (for example people and their handouts) can be derived from the concepts explicated at levels two, three, and four. While less obvious, missing concepts also can be explicated for the first conceptual model where no notion of the self or identity is explicated. As an observation of human phenomenon, MIC would suggest that the conceptualizations at the other three levels entail some notion of identity, which has thus far, remained implicit in our conceptualization. For now, we might suggest that it is a component of human self determined, to a significant extent, by social structure. This is evidenced by the fact that the respondent's emotional fatigue was a level three outcome of the cyclical process where individual addiction and addiction treatment is embedded in the social structure of poverty. Given the brevity of our excerpt, these imputations can only be tentatively postulated, but the MIC generator itself gives rise to these sorts of possibilities and comparison with other data would clarify them.

As a system of logic, the MIC generator acts like a de-fragmenting program on a computer. Without a logic structure, we have no way to systematically transform a pool of independent concepts into conceptual models. By placing a concept into the generator and then working with its inherent logic, the data immediately begins to take structure. For example, if we take the single concept of 'embarrassment' from the excerpt and place it at level three of the MIC generator, as it is clearly a concept of feeling, we are immediately compelled to ask how this feeling is produced, a question that focuses on the dynamic level.

\section{PROBLEMATIC TWO: CONCEPTUAL ABSTRACTION}

Grounded theory attempts not only to build conceptual models specific to particular pieces of data, but for broader contexts. The above excerpt would not be terribly significant if it stood alone; a researcher likely would not wish to delineate conceptual structures, which explain only one respondent's homeless experience. Building more general conceptual models requires making connections throughout one's data, delineating concepts and concept structures 
TABLE 3. Fractal concept model of homeless service provision

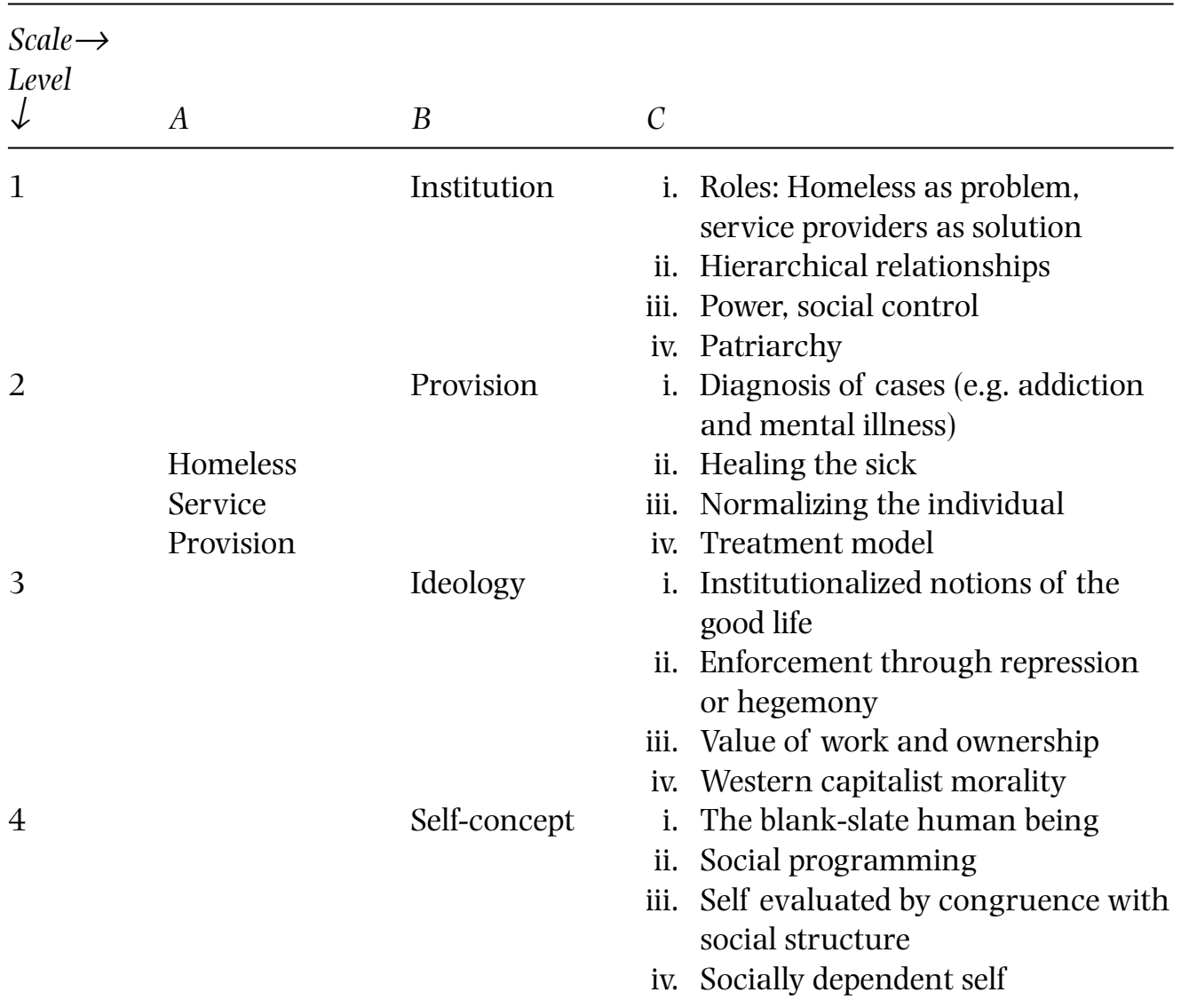

Note: 1/i - Static; $2 / \mathrm{ii}$-Dynamic; 3/iii - Evaluative; 4/iv - Self/Identity

which hold for multiple respondents and which theoretically will hold for those not sampled (although many assert that verification of emergent models is not the aim of grounded theory, e.g. Glaser, 2002). So if theory construction is to have any real significance, if it is to suggest concepts and concept structures, which extend beyond specific sets of observations of particular respondents, it must have a systematic way of abstracting broader concepts and conceptual models from those specific to the data.

Just as using the MIC generator gives a systematic way of delineating the relationships of conceptual pieces, the self-similar structure of fractal analysis and the notion of catiteration suggests a way to proceed with generalization. In fractals, iteration works toward complexity, for example, when the fractal tree branches. But we also can work backwards toward less complex structures through the process of catiteration. This is, in fact, how Mandlebrot made his initial discovery (see Mandlebrot and Hudson, 2004). For all of the various incarnations of grounded theory, all would agree that initially it is data specific observations that yield insight into more general concepts (though this need not imply a simple grouping process as discussed earlier). However, fractals 
yield a crucial addition. Catiteration for fractal concept analysis is a process of abstraction, but also one of uncovering self-similar structures. When read from right to left, Table 3 shows just such a process (please note that for logistical reasons the lower levels are not illustrated as contained within the higher levels, as in Table 1).

While we employ our homeless research here only as an illustration, some general substantive information is a necessary backdrop. Most work on homelessness is strikingly similar in that nearly all reaches the same vague bifurcated solution: 1) offer more services to the individual homeless; and 2) address inadequacies of social structures which predicate homelessness (see Shlay and Rossi [1992] for a meta-analysis of homeless literature). Since homeless services routinely are postured as at least one part of this solution, there is a dearth of work critically examining the model on which those services are delivered, their implicit goals and assumptions, and the ways in which service institutions financially and ideologically are vested in those same inadequate social structures which predicate homelessness (for an exception see Lyon-Callo [2000] and Hopper [2003]). This is not an easy stance to take because it compels criticism of some very well-meaning and good-hearted people, but it is as important as it is difficult.

The model in Table 3 emerged from our ethnographic research on the street homeless, during which we were compelled to assess their complaints of local homeless shelters by incorporating those shelters and their clients into our sampling frame. What emerged can generally be understood as part of the broader phenomenon of the medicalization of homelessness, a theme we later found addressed in a small corner of the homeless literature (see Hopper, 2003; Lyon-Callo, 2000; Mathieu, 1993; Snow et al., 1986).

Work addressing medicalization of homelessness typically focuses on its conceptual conflation with mental illness and addiction. From his ethnographic work in a homeless shelter, Lyon-Callo (2000: 330) writes, '...focus on "disease" within the discourses of "helping" actually obliterates discussion of alternative explanations and thus hinders developments aimed at resolving homelessness through altering class, race or gender dynamics.' His narrative exposition of this point, and his observations of specific shelter practices which reflect it, yield wonderful insights.

However, while Lyon-Callo (2000) portends connections between the medical paradigm and broader cultural contexts in which it may be embedded, there is no explicit tie between his shelter-specific observations and these broader notions. Emergent themes about homeless services from our work are a substantive complement to Lyon-Callo (2000), but using fractal concept analysis we show such between-scale linkages, i.e. how a systematic path can be opened between specific observations of medicalization within homeless shelters and the broader cultural contexts of which they are an instance.

In column $\mathrm{C}$ emergent themes are organized according to the logic of the MIC generator. Some of these emerged directly from the data; some were 
uncovered in the memo writing process using the schema of the MIC generator. During the coding process we began to pick up on the notion that the homeless were routinely postured as 'the problem'. In opposition, homeless services were routinely postured as 'the solution' (1C-i), sometimes explicitly and other times by the various ways they were held in contradistinction to 'the problem'. These are not benign role definitions, but rather predicate an entire course of action. These static conceptualizations engender only one possible dynamic relationship between the two groups (1C-ii). That is, the solution always stands above the problem in a relationship. The question becomes why is this the case, i.e. what value does it serve to posture the service providers above the homeless in an institutional structure? The answer is that it gives service providers power (1C-iii). We can then recognize the identity of such an organization as patriarchal (1C-iv).

All of these conceptual themes are embedded in the overall static paradigm of homeless services as an institutional structure. That is, these are the things that define the physical organization of the shelter. Just as, in the example above, we can understand pediatrics as relatively feminine in the context of the masculine physician profession (Salzinger, 2004), here we can understand that there are dynamic relationships, values, and an identity within the overall static concept of the institutional structure. Conceptual designations at one level of scale must be read in relation to the catiterated concepts in which they are embedded. The question of dynamics in row one, for example, is about the relationship of the static concepts, rather than about pure action; it is a dynamic concept embedded in an overall static concept.

In column $\mathrm{C}$ at level 2 we can see the MIC generator repeat itself, but now within the overall dynamic concept of service provision (which can now be more clearly postured against the service institution at the static level). Homeless service providers seek to diagnose homeless cases, most often as being either mentally ill or drug addicted (2C-i). These diagnoses not only resonate with popular conceptions of homelessness, but funding for services is often tied to treatment of these particular afflictions. Again, a particular course of action follows these static disease concepts where service providers engage in healing the sick (2C-ii), the success of which is judged by the extent to which the homeless person can be returned to a normal state, i.e. working and maintaining a residence and other possessions (2C-iii). At the identity level, we can name this as the treatment model (2C-iv).

For levels one and two together, we can see a conceptual delineation of the medical model of homeless service provision. Fractal concept analysis adds conceptual clarity in a variety of ways. First, we can understand that problems of the shelter are predicated by its structural organization and particularly by the general acceptance of problem/solution role designation. Freire (1994) notes that oppression is fundamentally a matter of the elites' ability to ontologically define the world of the oppressed. Here we can see a clear instance of this. When postured as 'the problem' the homeless are stripped of their 
subjectivity, transformed into objects, and therefore easily oppressed by the medical model, which specifies particular pathologies and then naturally seeks to treat them. With such definitions in play, what else would the service providers do? While clarification of institutional structure and the action of service provision is important, we can use fractal analysis to extend further into cultural context. We have identified the medical model of homeless service provision in terms of its structure and action. While we can see specific values naturally built into the structure and dynamics of service provision, fractal analysis allows us to look for intrinsically valuative structures which function in relation to the static and dynamic components of service provision (see column B).

At level two in column B, we can see that the diagnosis and healing dynamics are not purely matters of object and action, but are guided by a particular valuative notion of 'normality' (2C-iii), a notion not coincidentally central to Parson's (1951) sick role. At level three (column B) we can ask what sorts of values guide both the overall concepts of structure and service provision of homeless shelters. As institutions, homeless services employ institutionalized notions of normality, of success. That is, the homeless person is guided toward presupposed goals, what we might call institutionalized notions of the good life (3C-i). As external to, and standing above the individual, rather than emerging from their own worldview, institutionalized values are implemented through hegemonic persuasion and the repression of the punishment paradigm (3C-ii). ${ }^{12}$ The specific values institutionalized in homeless services reflect wider cultural notions such as the value of work and ownership (3C-iii). Here we can see exactly how western capitalist value structures stand in relation to structural arrangement and treatment dynamic of homeless shelters to legitimate such a paradigm. At the identity level (3C-iv), we can simply refer to this value structure as western capitalist morality, a value system which stands above individual human beings (this generally is the feminist critique of western culture and classic moral philosophy in particular, see Gilligan, 1982), and presupposes and implements moral qualities of work and ownership (see Sahlins [1972] for discussion on such values as distinctly cultural products).

We can understand the catiterated value of the model (column B) as qualitatively different than the iterated values in the overall static and dynamic levels (1C-iii and 2C-iii). The iterated values in levels one and two can be stated as the specific values of the organizational schema and the treatment dynamic. But these do not provide any explanation of why such an organizational schema or treatment dynamic are selected out of the variety of other possibilities. The overall value concept at level three (column B) gives us this explanation. The patriarchal structure and the medical treatment model are chosen because they fall in line with broader cultural values of western capitalism, the protestant ethic.

While within the other MIC levels, we can locate identities for the iterated conceptual complexes (1C-iv, 2C-iv, 3C-iv), At level four column B, our analysis 
calls us to ask what conception of the human self is endemic to the overall conceptual model of homeless service provision. Just as the structure and action of the service model is logically consistent with broader cultural values, all of these are logically consistent with the dominant western concept of the self, the tabula rasa (4C-i). The social programming dynamic (4C-ii) naturally emerges when we define the human being fundamentally as a blank slate, a vessel to be filled with knowledge. The sick, misguided, or morally corrupt homeless person, as a product of socialization, can only be fixed with more socialization. Here again, socialization is not a benign process, but rather works toward particular valuative goals. Specifically, for the fourth level, it evaluates on the congruence between self and society (4C-iii). Of course, taking a critical perspective, and noting social structural contributions to homelessness, we might question whether it is in fact a healthy individual who does not fit in a sick social structure. At $4 \mathrm{C}$-iv we can see that the tabula rasa definition of self postures the human being as wholly dependent on society. While sociology has no consensus about the relationship of the individual and society, and much work tends to see social structure as largely dominant, few would be comfortable taking the extremist position highlighted here.

In column B we see a catiteration of the four MIC models iterated in column C. Indeed we can understand homeless service provision as a conceptual complex which entails a structural organization (static), engages in treatment of the homeless (dynamic), entails particular values of working and ownership (value), and which employ a particular ontological concept of the human being (self/identity), which makes possible a social programming model. The specific themes of column $\mathrm{C}$ follow inherently the logic of the MIC generator, but those four models together also follow the same logic. We can catiterate to column A to say that it is a model of homeless service provision, which begs the question about where homeless services stand in relation to other social institutions. While it is only speculation here, as an institution fundamentally about the execution of the treatment model, i.e. action, we suspect that it can be catiterated to an overall dynamic level, when postured in relation to other social institutions.

Through our exposition of the fractal model in figure seven, one can see that fractal concept analysis allows a systematic process of abstraction because catiteration follows the MIC logic structure across levels of scale. Further, using fractal concept analysis could potentially yield cross-study comparisons, where, for example, the conceptual relationships between homeless service institutions and others are analysed, the question brought forth by our speculated catiteration to column A.

\section{Justifying synthesis}

We expect that some grounded theory proponents will be skeptical of our suggestions since it often is claimed that grounded theory rejects any a priori 
conceptual designations (while Glaser [2002] is adamant about this point, his 1978 coding families suggest that there is room for heuristic formulations like our MIC generator). If indeed all concepts must emerge from the data and fractal concept analysis employs concepts independent of the data, particularly where we offer the MIC structure as a generator, we must offer some justification.

It is overly simplistic to assert that all concepts must emerge from the data. Wacquant (2002: 1481) correctly has called this, 'the epistemological fairytale of grounded theory.' Clearly none of us can actually be free of all concepts at the start of a data analysis (see also Dey, 1999). At the very least we must hold some concept of 'data' in order to begin analysis. Rather than clinging hopelessly to such a rigid and overly-simplistic rule of 'no concepts,' we must take a more sophisticated view and examine which kinds of concepts are appropriate and which are not. Answering this question is not simply a matter of identifying meager nuances, but will determine the future success of grounded theory in a discipline with other methodological techniques that are evolving alongside increasingly sophisticated epistemologies, although often unconsciously. Take for example Hierarchical Linear Modeling which implicitly recognizes change (slope) as a variable in and of itself, rather than as only an outcome measure of relation between two variables. To borrow from our MIC language, we can understand this to be, at least implicitly, the recognition of fully dynamic concepts, those with ontological status beyond static variables, the units which are most common to standard linear models.

The ontological categories proposed by MIC are not the same as the substantive concepts that grounded theory suspends in advance. The reader will note from Table 2 that using MIC epistemology does not affect the substantive content of the concepts used to define the data. Rather, when interpolated onto the substantive codes it simply marks additional ontological information by delineating what kind of concept it is and its relationship with other concepts through the logic inherent to the MIC generator. What grounded theory rightly opposes is approaching the data a priori with substantive concepts (what we might call content concepts). For example, if we had approached the coding of our excerpt (Table 2) with the often presupposed concepts of mental illness or addiction, and moreover with a notion of these as casual rather than symptomatic, we surely would have violated the premise of grounded theory. However, MIC does not provide any such content, but rather denotes a logical concept form, and especially forces theorists to consider all possible conceptual forms and to make a conscious decision on which ontological quality (static, dynamic, etc.) is most appropriate. The researcher is still able to approach MIC forms without a priori conceptual substance.

Moreover, since fractals utilize an internal logic, nothing about fractal concept analysis presupposes any particular universal logic structure. In fact, the insufficiency of such a presupposition is implied in our critique of routinely applying linear logic to human life. Rather we can see our choice of the MIC generator as rooted in the data. As MIC denotes a particularly human logic 
structure, we can say that the MIC generator, as the most basic component of our analysis, 'emerges' from our most basic observation of the data, that it is data about human beings. If E.O. Wilson had approached his study of ants $a$ priori with the MIC generator then he would perhaps have violated the premise of emergence. We have not. In addition, as fractals are self-organized systems, we can assert the benefit of the MIC generator for human data without wholesale denial of other possible generators. In other words, we can move qualitative research away from relativism, without moving toward naïve positivism, through the fractal notion of self-organized systems. This allows logical observation without necessitating any particular logic which may not work well for given systems.

Glaser and Strauss (1967) originally suggested that grounded theory be evaluated by how well it works. Fractal concept analysis provides a way for the researcher to systematically abstract from the data and perceive structural relationships between concepts. Since building theory is by definition dependent on these abstractions and specifying conceptual relations, and the purpose of the grounded theory technique is to build theory, fractal concept analysis clearly is of benefit.

NOTES

1. The name 'fractal concept analysis' and some of our particular insights into using fractals for social science indeed are novel. However, we draw here from the foundational and revolutionary work of Andrew Abbott (2001). Moreover, we are neither the first to build directly off Abbott's work (see Salzinger, 2004) nor to recognize the general potential for utilizing fractals in social science (see Agar, 2004; Kuhn and Woog, 2005; Ward, 1995).

2. See Charmaz (2000) for a critique of the debates surrounding grounded theory, and (2006) for the historical context of the emergence of the grounded theory method. Dey (1999) also provides a worthy look back at the contested history of this method. Rennie (1996) gives an in-depth treatment of the epistemological underpinning of the divergent approaches of Glaser and Strauss.

3. Glaser (discovery) and Strauss (verification) eventually moved in somewhat divergent directions with their grounded theory method (see Corbin and Strauss, 1990; Rennie, 1996; Strauss, 1987; and Strauss and Corbin, 1998).

4. Open-ended means suspending preconceived concepts when coding, albeit, Dey (1999: 251) points out that, 'there is a difference between an open mind and an empty head.' We discuss this in more detail later. Also, we prefer a line by line start, but initial coding can be word by word, or incident by incident depending on the type of data.

5. Glaser and Strauss (1967) further suggest that this process of continually synthesizing data and concepts ought to be instructive for one's own work, as well as guiding interaction between professionals. They write, 'If each debunker thought about the potential value of comparative analysis, instead of satisfying his urge to "put down" a colleague, he would realize that he has merely posed another comparative datum for generating another theoretical property or category' (Glaser and Strauss, 1967: 22). Clearly we agree as this is the very thrust of the synthesis of grounded theory and fractal concept analysis which we present here. 
6. Strauss (1987) and Strauss and Corbin (1998) suggest axial, as a third type of coding. They see initial coding as the breaking down and axial coding as the putting back together (see also Corbin and Strauss, 2008). Similarly Clarke (2005) sees axial coding as elaborating coding categories so they can be integratively diagrammed. Glaser (1992) argued that there was no need for axial coding, as the theoretical coding families he included in his earlier work "weaved the fractured story back together' (Glaser, 1978: 72).

7. Again, we are aware that Glaser (2002) particularly objects to pigeon holing grounded theory as a qualitative method, but aside from its broader potential, its actual impact clearly has been on qualitative research.

8. We should note that this is not the thrust of Salzinger's (2004) piece, which is a fractal analysis of gendered identities in Mexican factories. Her medical professions example, however, is a more widely recognizable context and we thus borrow it for our explanation, with some addition of our own to the nursing side of the fractal which we derive from the Men in Nursing Survey (Hodes Research, 2005).

9. This echoes generally the feminist critique of science (see Harding, 1991, 1998).

10. We are aware that we may appear to violate the basic epistemological premise of grounded theory by bringing an a priori framework to bear on observations of data. Glaser (2002) is particularly bothered by this type of corruption to grounded theory. However, we ask for temporary indulgence on this point, which we will address below.

11. It is philosophically and personally important for the authors to note that this does not compromise arguments for the ethical treatment of animals, which can be stated in our framework as giving moral standing to those beings who have level three experience, while still recognizing something distinctive about human life vis-a-vis non-human animals.

12. Lyon-Callo (2000) well documents the consequences of not accepting the treatment regimen of the shelter.

REFERENCES

Abbott, A. (2001) Chaos of Disciplines. Chicago, IL: University of Chicago Press.

Agar, M. (2004) 'We Have Met the Other and We're All Nonlinear: Ethnography as a Nonlinear Dynamic System', Complexity 10: 16-24.

Bryant, A. (2002) 'Re-Grounding Grounded Theory', Journal of Information Technology Theory and Application 41(1): 25-42.

Bryant, A. and Charmaz, K. (2007) Handbook of Grounded Theory. Thousand Oaks, CA: Sage.

Carrithers, M. (1990) 'Is Anthropology Art or Science?' Current Anthropology 31: 263-72.

Charmaz, K. (2000) 'Grounded Theory: Objectivist and Constructivist Methods', in N.K. Denzin and Y.S. Lincoln (eds), Handbook of Qualitative Research, pp. 509-35. Thousand Oaks, CA: Sage.

Charmaz, K. (2001) 'Grounded Theory in Ethnography', in P. Atkinson, A. Coffey, S. Delamont, J. Lofland and L. Lofland (eds), Handbook of Ethnography, pp. 160-74. Thousand Oaks, CA: Sage.

Charmaz, K. (2006) Constructing Grounded Theory: A Practical Guide Through Qualitative Analysis. Thousand Oaks, CA: Sage.

Clarke, A.E. (2005) Situational Analysis: Grounded Theory After the Postmodern Turn. Thousand Oaks, CA: Sage. 
Corbin, J. and Strauss, A.L. (1990) 'Grounded Theory Research: Procedures, Canons, and Evaluative Criteria', Qualitative Sociology 13(1): 3-21.

Corbin, J. and Strauss, A.L. (2008) Basics of Qualitative Research: Techniques and Procedures for Developing Grounded Theory (3rd Ed). Thousand Oaks, CA: Sage.

Dey, I. (1999) Grounding Grounded Theory: Guidelines for Qualitative Inquiry. San Diego, CA: Academic Press.

Freire, P. (1994) Pedagogy of the Oppressed. New York: Continuum.

Gilligan, C. (1982) In a Different Voice: Psychological Theory and Women's Development. Cambridge, MA: Harvard University Press.

Glaser, B.G. (1978) Theoretical Sensitivity. Mill Valley, CA: The Sociology Press.

Glaser, B.G. (1992) Basics of Grounded Theory Analysis: Emergence vs. Forcing. Mill Valley, CA: Sociology Press.

Glaser, B.G. (2002) 'Conceptualization: On Theory and Theorizing using Grounded Theory', International Journal of Qualitative Methods 1, URL (consulted 31 October 2005): http://www.ualberta.ca/ ijqm/

Glaser, B.G. and Strauss, A.L. (1965) Awareness of Dying. Chicago, IL: Aldine.

Glaser, B.G. and Strauss, A.L. (1967) The Discovery of Grounded Theory: Strategies for Qualitative Research. Chicago, IL: Aldine.

Glaser, B.G. and Strauss, A.L. (1968) Time for Dying. Chicago, IL: Aldine.

Harding, S. (1991) Whose Knowledge: Whose Science: Thinking from Women's Lives. Ithaca, NY: Cornell University Press.

Harding, S. (1998) Is Science Multicultural?: Postcolonialisms, Feminisms, and Epistemologies. Bloomington, IN: Indiana University Press.

Hodes Research (2005) 'Men in Nursing Survey', URL (consulted 10 June 2007): http://www.hodes.com

Hopper, K. (2003) Reckoning with the Homeless. Ithaca, NY: Cornell University Press.

Hume, D. (1739 [1978]) A Treatise of Human Nature ( L.A. Selby-Bigge, ed.; P.H. Nidditch, rev.). Oxford: Oxford University Press.

Kuhn, L. and Woog, R. (2005) 'Vortical Postmodern Ethnography: Introducing a Complexity Approach to Systemic Social Theorizing', Systems Research and Behavior Science 22: 139-50.

Kuhn, T.S. (1962) The Structure of Scientific Revolutions. Chicago, IL: University of Chicago Press.

Lyon-Callo, V. (2000) 'Medicalizing Homelessness: The Production of Self-Blame and Self-Governing within Homeless Shelters', Medical Anthropology Quarterly 14(3): $328-45$.

Mandelbrot, B.B. (1982) The Fractal Geometry of Nature. New York: W.H. Freeman and Company.

Mandelbrot, B.B. and Hudson, R.L. (2004) The (Mis)Behavior of Markets: A Fractal View of Risk, Ruin \& Reward. New York: Basic Books.

Mathieu, A. (1993) 'The Medicalization of Homelessness and the Theater of Repression', Medical Anthropology Quarterly 7: 170-84.

Parsons, T. (1951) The Social System. New York: The Free Press.

Popper, K.R. (2002) The Logic of Scientific Discovery. London: Routledge.

Rennie, D.L. (1996) 'Grounded Theory Methodology: The Pressing Need for a Coherent Logic of Justification', Theory and Psychology 8: 101-19.

Rennie, D.L. (1998) 'Grounded Theory Methodology: Reconciling Realism and Relativism', Theory and Psychology 10: 481-502.

Sahlins, M.D. (1972) Stone Age Economics. New York: Rinehart. 
Salzinger, L. (2004) 'Revealing the Unmarked: Finding Masculinity in a Global Factory', Ethnography 5: 5-27.

Shlay, A.B. and Rossi, P.H. (1992) 'Social Science Research and Contemporary Studies of Homelessness', Annual Review of Sociology 18: 129-60.

Snow, D.A., Baker, S.G., Anderson, L. and Martin, M. (1986) 'The Myth of Pervasive Mental Illness Among the Homeless', Social Problems 33(5): 407-23.

Strauss, A.L. (1987) Qualitative Analysis for Social Scientists. New York: Cambridge University Press.

Strauss, A.L. and Corbin, J. (1998) Basics of Qualitative Research: Grounded Theory Procedures and Techniques. Thousand Oaks, CA: Sage.

Wacquant, L. (2002) 'Scrutinizing the Street: Poverty, Morality, and the Pitfalls of Urban Ethnography', American Journal of Sociology 107: 1468-1532.

Wasserman, J.A. and Wilson, K.L. (2008) 'Insight on Demand: Teaching Social Science Using Heart-thinking Analysis of Visual Imagery', Journal of Curriculum and Pedagogy 5: $134-48$.

Wundt, W. (1973) An Introduction to Psychology. New York: Arno Press.

Wilson, K.L. and Lowndes, F. (2004) 'Heart-Thinking: An Archetypal Epistemology for the Humanities and the Sciences', International Journal of the Humanities 1(1): 221-31.

Wilson, K.L., Wasserman, J.A. and Lowndes, F. (2008) 'Saving Society from Instrumental Rationality: A Study of Mahler and Homeless Research in the Spirit of Adorno's Critical Musicology', Journal of Music and Meaning 7/Fall/Winter.

Wilson, K.L., Wasserman, J.A. and Lowndes, F. (2009) 'Picture and Social Concept: Fractal-Concept Analysis of Advertising Art', Visual Communication: Forthcoming.

JASON ADAM WASSERMAN is currently an Assistant Professor of Sociology at Texas Tech University where he also serves as Director of the Community and Urban Studies program. His interests include the homelessness, concepts of self, qualitative methods, medicalization, bioethics, and ethnography. Address: Department of Sociology, Anthropology, and Social Work, Texas Tech University, MS41012, Holden Hall 158, Lubbock, TX 79409-1012, USA. [email: jason.wasserman@ttu.edu]

JEFFREY MICHAEL CLAIR is Associate Professor of Sociology at the University of Alabama at Birmingham. His areas of specialization are social medicine, homelessness, social psychology, ethnography, and death and dying. Address: Department of Sociology, University of Alabama at Birmingham, Heritage Hall 460, 1530 3rd Ave S., Birmingham, AL, 35294, USA. [email: jclair@uab.edu]

KENNETH L. WILSON is Associate Professor at the University of Alabama at Birmingham. His current research interests center on the use of fractals, chaos theory, and Deleuzian postmodernism for developing new views of epistemology that bridge science and the arts. Address: Department of Sociology, University of Alabama at Birmingham, Heritage Hall 460, 1530 3rd Ave S., Birmingham, AL, 35294, USA. [email: knwilson@uab.edu] 